\title{
Impact of COVID-19 on post graduate medical education and training
}

\author{
Sharon C. Enujioke ${ }^{1 *}$, Kimberly McBrayer ${ }^{1}$, Katherine C. Soe ${ }^{2,3}$, Teresa M. Imburgia ${ }^{1,4}$ and Cynthia Robbins ${ }^{1}$
}

\begin{abstract}
Purpose: The novel Coronavirus Disease 19 (COVID-19) has had a significant impact worldwide that led to changes in healthcare. The purpose of this study was to evaluate the effect of the COVID-19 pandemic on trainee's mental health and educational preparedness.

Methods: Trainees at the Indiana University School of Medicine were surveyed regarding the impact of the COVID-19 pandemic on their training. Using a Likert scale, participants were asked questions pertaining to educational preparedness, mental health, and clinical work during the pandemic. Data was analyzed using SPSS version 27. The study was approved as exempt by the Institutional review Board (IRB).

Results: 324 of the 1204 trainees responded to the survey. The respondents were $76 \%$ white with an equal distribution of males and females. A majority of the respondents were first year residents with an equal distribution of second, third, and fourth year residents. Twenty-three percent of respondents were in a procedural residency or fellowship program. Better perceived educational preparedness was associated with an improved home-work balance during COVID-19 $(\beta=0.506, p<0.0001)$ and having a department that advocated/supported focus on mental health during COVID-19 $(\beta=0.177, p<0.0001)$. Worse perceived educational preparedness was associated with being in procedural vs. non-procedural dominant training program $(\beta=-0.122, p=0.01)$.
\end{abstract}

Conclusion: COVID-19 has had a significant impact on the training experience of residents and fellows. Departmental support increased mental well-being and perceived education preparedness in trainees. Trainees that felt they had a better home-work life balance had better educational preparedness compared to their peers. Also, trainees in procedural programs had less educational preparedness compared to their peers in non-procedural programs. This study highlights the importance for programs to find avenues to increase educational preparedness in their trainees while being attuned to the mental health of their trainees.

\section{Introduction}

The novel Coronavirus Disease 19(COVID-19) has had a significant impact on health care systems around the world. The COVID-19 pandemic's effect on resident physicians and fellows in training remains limited. Studies of residents during this time have examined the use of

\footnotetext{
*Correspondence: senujioke@hotmail.com

${ }^{1}$ Division of Adolescent Medicine, Department of Pediatrics, Indiana University School of Medicine, 410 West 10th Street. Suite 1001, Indianapolis, IN 46202, USA

Full list of author information is available at the end of the article
}

telemedicine as a means of promoting safety while maintaining access and minimizing disruption in clinical care. Many innovative solutions have arisen in response to medical and surgical education changes, including virtual learning, videoconferencing, and social media [1]. However, these have also highlighted limitations with regard to rapport development with patients, privacy and confidentiality, and access to technology [2].

One study of resident mental health at a large academic medical center revealed significantly higher rates of reported stress and burnout in COVID-19 exposed physician trainees compared to non-exposed. Female 
and unmarried trainees demonstrated higher rates of stress and depression, respectively [3]. In fact, over half of resident trainees in Brooklyn, New York expressed anxiety for at least several days over a two week period [4]. Despite decrease in operating room (OR) time, a survey of otolaryngologists during the COVID-19 pandemic found that nearly half reported anxiety, depression, distress and burnout, with a greater prevalence in residents (compared to attendings), females, and those in states with over 20,000 positive cases [5]. Together, these studies reflect a common theme: the importance of being mindful of and supporting the mental health of frontline healthcare workers, including physician trainees.

To our knowledge, this is the first study to assess the perceived impact of the COVID-19 pandemic on residents' and fellows' clinical training and mental health support across multiple subspecialties at a large medical institution. This study aims to describe the impact of COVID-19 on post-graduate medical trainees and investigate what was associated with positive educational preparedness during COVID-19.

According to the Indiana State Department of health, there have been over $672 \mathrm{~K}$ COVID cases with $12 \mathrm{~K}$ deaths in Indiana, representing the thirteenth highest death rate in the country [6]. The highest rate of COVID admissions and deaths occurred in December of $2020[7,8]$. Indiana University Health (IUH) is the largest network of physicians and hospitals in the state. IUH partners with the Indiana University School of Medicine (IUSOM), which sponsors 100 ACGME-accredited residency and fellowship programs. In 2021, there were 985 residents and 219 fellows associated with IUH and IUSOM $[9,10]$. The COVID vaccine was available to all house staff at the time of this survey.

\section{Methods}

\section{Participants}

Participants were sampled from the 1204 residents and fellows associated with IU School of Medicine. They received an emailed survey to complete. This represented all residents and fellows across all programs at IUSOM.

\section{Materials}

Participants were asked demographic data, overall impression of training during COVID-19 and questions pertaining to educational preparedness, clinical work, and mental health during the pandemic. Participants could leave comments on the final question. Gender, race, marital status, whether they had children living at home, and COVID-19 exposure and if they acquired the infection status were assessed. Overall impression included questions such as "COVID-19 has had a positive impact on my training." Educational preparedness included "I have had more time for self-directed study during COVID-19" and "I feel more prepared for my Inservice training exam (ITE)/boards." Questions were measured on a Likert type scale of 1 through 5 , with 1 being strongly disagree and 5 being strongly agree.

\section{Procedure}

The survey was implemented via a web-based platform (Redcap) and completed by means of voluntary participation. Surveys were distributed by email through the Graduate Medical Education (GME) office at the beginning of March 2021 and re-sent three weeks later to all trainees. The team then reached out to program directors to email the survey to their trainees. After a month of collecting responses, the survey was closed for analysis of results. The individual email addresses were not known by the researchers, and data was de-identified by Redcap.

Data analysis was performed with SPSS version 27 for Windows (IBM, Armonk NY). Frequency data are presented on demographics and COVID-19 impact. Due to lack of power, transgender individuals were coded with their associated gender (e.g. transgender females coded with cis-gender females) and two non-binary individuals were coded as missing for the purpose of analysis involving gender. Racial data was analyzed both with individual races and with races grouped as white and non-white. Fifty-two different resident and fellow groups answered the survey, and these were grouped into procedural (determined as all procedure-dominated residencies and fellowships) and non-procedural programs for added power. Procedural program included all surgical specialties and subspecialties as well as anesthesiology, dermatology, and pediatric dentistry.

Five educational items were combined into an educational preparedness scale (Chonbach's alpha $=0.61$ ) to assess what items were associated with a better perceived educational preparedness during COVID-19. The study was approved as exempt by the Indiana University Institutional Review Board (IRB).

\section{Statistical analysis}

Bi-variate associations of candidate predictors with perceived educational preparedness were analyzed using t-tests and chi-squared tests. Predictors were then entered into a multivariable linear regression model.

\section{Results}

The survey was sent to the 1204 trainees at IUSOM and 324 completed the survey. Of the respondents, $48 \%$ identified as female and $76 \%$ as white. Sixty-four percent identified having a current partner, and $20 \%$ had at least one child under the age of 18 . Mean years post-graduate were 2.9 (1.6), with a majority of the respondents identifying as 
first year residents and an equal distribution of the second through fourth year residents. Twenty-three percent of respondents identified as being involved in a procedural residency, which was classified as a procedural dominant specialty or fellowship program. Positive COVID-19 testing in the trainee or their immediate household was $15 \% ; 31 \%$ of respondents endorsed having to quarantine at least once. Mean answer for the Educational preparedness domain questions was $3.0(0.7)$. Table 1 provides demographic information and Table 2 provides the mean scores of overall COVID impact, educational preparedness, clinical questions, and mental health items. Table 3 provides bivariate analysis involving education preparedness questions compared to demographic data and mental health questions, which yielded significant results for female gender ( 3.1 vs. 2.9 for males, $p=0.001$ ), number of years post-graduate $(p<0.0001)$, no kids ( $3.1 \mathrm{vs.} 2.8$ for those with kids, $p=0.003$ ), and departmental support of mental health $(2.2,0.9, \mathrm{p}<0.0001)$. Multivariable analysis for educational preparedness was performed using linear regression (see Table 3 ). The adjusted $\mathrm{R}^{2}$ for this model was 0.364 . Better perceived educational preparedness was associated with an improved home-work balance during COVID-19 $(\beta=0.506, p<0.0001)$ and having a department that advocated/supported focus on mental health during COVID-19 $(\beta=0.177, \mathrm{p}<0.0001)$. Worse perceived educational preparedness was associated with being in procedural vs. non-procedural training program $(\beta=-0.122, p=0.01)$.

\section{Discussion}

This study adds to a growing body of literature on the impact of the COVID pandemic on physicians in training (resident physicians). The COVID pandemic created unique challenges for all of healthcare, and many institutions rely on physicians in training to staff hospitals and

Table 1 Sample characteristics $(N=324)$

\begin{tabular}{ll}
\hline & Mean, SD or $\mathbf{n}, \%$ \\
\hline Gender female & $150,47.5 \%$ \\
Race white & $221,75.9 \%$ \\
Has a current partner & $204,63.7 \%$ \\
Procedural dominated programs* & $67,20.6 \%$ \\
Has Kids & $66,20.4 \%$ \\
First year resident & $85,26.3 \%$ \\
Number of years post grad & $2.9,1.6$ \\
You or someone in your immediate household & $50,15.4 \%$ \\
tested positive for COVID & \\
Quarantined for COVID contact & $100,30.9 \%$
\end{tabular}

*General surgery, orthopedic surgery, anesthesia, dermatology, otolaryngology, OBGYN, Urology, OMFS, peds dentistry, urogynecology, transplant nephrology, uro-endourology, neurosurgery, ophthalmology, peds urology, peds cardiac anesthesia
Table 2 COVID Impact on education, clinical skills, and mental health

COVID Items ( $1=$ strongly disagree $-5=$ strongly agree $)$ Mean, SD

\section{Overall COVID attitude}

COVID has had a Positive Impact on training

$3.56,1.1$

COVID has had a Negative Impact on training

$2.02,0.82$

Educational Attainment (5 items, $a=0.641$ )

3.0, 0.7

More time for self-directed study

$2.95,1.11$

Felt prepared for ITE

$3.54,0.85$

Zoom lectures were effective

$3.44,1.29$

Preparedness to practice

$2.31,0.97$

I have had time to read

$2.78,0.91$

\section{Clinical Items}

Efficient at research

$2.95,0.99$

Clinical skills not impacted by COVID

$3.13,1.14$

Have time to care for patients

$2.99,0.92$

\section{Mental Health Items}

Improved life-work balance

$3.60,1.10$

Had to focus more on taking care of mental health

Department advocated/supported focus on mental health

Able to cope with changes

$2.75,0.99$

clinical programs, especially during times of high clinical volumes. The pandemic also created unique learning opportunities, with some disciplines more impacted than others based on the needs of COVID patients. Our survey found a relationship between better work life balance and positive education preparedness during the pandemic. Resident physician wellness is increasingly emphasized as a priority for training programs and mandated by the ACGME common program requirements; quality of life factors like sleep and social connection as well as educational autonomy and competence building have been found to be related to wellness [11].

Residency programs should have firm protections built in their design to protect quality of life which withstand stresses put on their clinical programs. Wellness positively impacts educational preparedness. Other studies have found preserving safety (personal protective equipment access and protection against contracting the virus) and maintaining high quality education can impact resident stress, so these are important areas to focus on for wellness. These studies also found transparency in program changes was also related to resident stress, and resident input into program changes should also be employed [12-15].

Our results also suggest that an individual's perceived departmental mental health support significantly correlated with educational preparedness during the COVID19 pandemic. Although previous studies have examined 
Table 3 Bivariate and Multivariate Analysis for Educational Attainment

\begin{tabular}{|c|c|c|c|c|c|}
\hline & $\begin{array}{l}\text { Educational attainment } \\
\text { Mean }\end{array}$ & Bivariate Beta & $P$ value & $\begin{array}{l}\text { Multivariable } \\
\text { Standardized Beta }\end{array}$ & $P$ value \\
\hline \multicolumn{6}{|l|}{ Gender: } \\
\hline Female & 3.1 & 0.177 & 0.002 & 1.589 & 0.068 \\
\hline Male & 2.9 & & & & \\
\hline \multicolumn{6}{|l|}{ Race: } \\
\hline White & 3.0 & 0.086 & 0.145 & 0.820 & 0.413 \\
\hline Non-white & 2.9 & & & & \\
\hline Current partner & 2.99 & -0.012 & 0.829 & 0.051 & 0.311 \\
\hline No current partner & 3.01 & & & & \\
\hline Surgical & 2.8 & -0.114 & 0.085 & -0.122 & 0.012 \\
\hline Non-surgical & 3.0 & & & & \\
\hline Has Kids & 2.8 & -0.164 & 0.003 & -0.089 & 0.085 \\
\hline No Kids & 3.1 & & & & \\
\hline Improved life-work balance. & - & 0.566 & $<0.0001$ & 0.506 & $<0.0001$ \\
\hline $\begin{array}{l}\text { Had to focus more on taking care of } \\
\text { mental health }\end{array}$ & - & -0.006 & 0.991 & 0.002 & 0.973 \\
\hline $\begin{array}{l}\text { Department advocated/supported } \\
\text { focus on mental health }\end{array}$ & - & 0.291 & $<0.0001$ & 0.177 & $<0.0001$ \\
\hline
\end{tabular}

$R^{2}=0.363$

mental health effects of the pandemic and changes in education for trainees, this is the first to our knowledge to report a correlation between these two variables. A systematic review examining challenges and innovative solutions for trainee education during the pandemic highlighted the rise in virtual solutions, including video conferencing platforms for both patient care and education, as a means of countering the limitations imposed by pandemic-related restrictions [1]. Principle challenges identified in the same review were largely related to the shortage of personal protective equipment (PPE) and drop in elective surgical cases. Both of these affect surgical residents more than non-surgical. This lack of PPE and reduction in resident-led elective surgery cases is likely to have affected perceived departmental support as well. A survey of urology residents across the United States further identified risk factors of worse mental health outcomes, which included perceived PPE access problems and susceptibility of household members, and local COVID-19 severity [16]. These studies both support and may help explain why surgical residents perceived a greater educational detriment during the pandemic than those who could utilize virtual means to assist with their assessments and management.

Notably, studies have consistently found that resident physician anxiety during this period often centered around loved ones' health over their own [16, 17]. One study found that the greater level of stress and burnout reported by those exposed to COVID-19 positive patients often included a cascade of worries about childcare and work-family balance [3]. Thus, it is reasonable that those who felt better supported in these domains would then have more reserve to engage in their education.

Recognizing the importance of frontline worker mental health, many programs both in and outside of the IU School of Medicine established new mental health access programs for healthcare provider assessments and therapy. Given that awareness is key to utilization, it would be interesting to investigate whether awareness of this program at the study institution affected perceived departmental support. Many programs also held virtual social gatherings in attempts to encourage social support systems. Telehealth peer support for frontline clinicians in the form of group and individual counseling facilitated by videoconferencing and phone, specifically regarding their work with COVID-19 patients, has been studied with reported utility in emotional processing, validation, and peer learning [18].

During the COVID-19 pandemic, residency and fellowships programs were faced with the task of restructuring their programs to protect trainees from contracting COVID-19 while maintaining their educational preparedness. While medical specialties rapidly evolved to telehealth visits, this could not replace the hands-on experience that procedure-dominated specialties glean from working in the operating room. This issue was reflected in our study findings, as non-procedural trainees reported higher levels of perceived educational preparedness compared to trainees in procedural programs during the study period. Despite reduction in 
Table 4 Key Recommendations

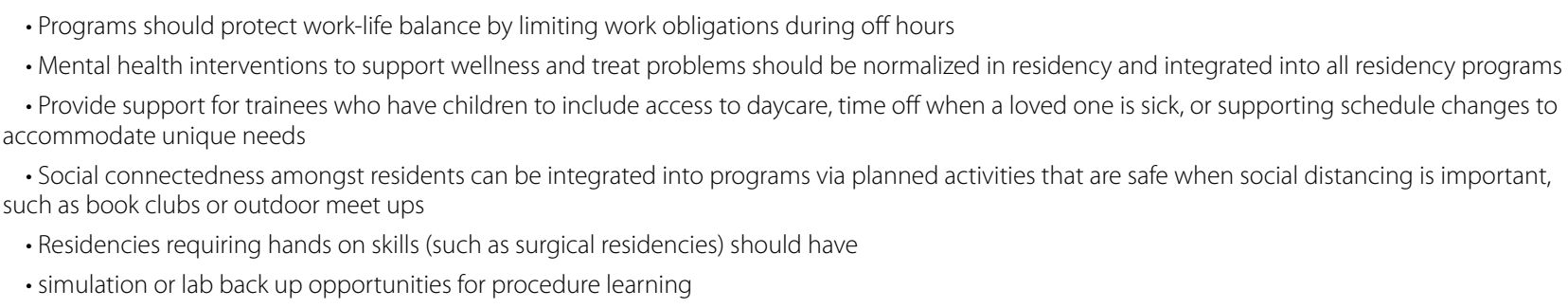

operating room time, surgical trainees reported increase in clinic and telemedicine visits leaving less room to study as well increased work burden when a fellow trainee was quarantined. Surgical residents had a significant decline in case volume during the pandemic. While the American Board of Surgery (ABS) modified graduation requirements, residents felt uncomfortable graduating with less than the traditional number of required cases. Similar to medical training programs, surgical programs adapted to COVID-19 by virtual didactics and telehealth visits. A few programs incorporated surgical simulators [19]. While telemedicine visits and didactics can offer some educational benefit, there is a push toward simulation training that replicates safety scenarios and targets training gaps due to the decreased workload during COVID-19 [20]. Looking at tools used during COVID19 pandemic to ensure trainees had optimal education preparedness at different programs will be imperative in planning for future pandemics. Key recommendations gleaned from our study are listed in Table 4.

\section{Limitations}

A limitation of this study was that specific components of well-being was not evaluated. Also, there was no preCOVID evaluation of training experience. Given the many training programs in the institution, we did not ask what changes had been implemented in each program to help with mental health during the COVID-19 pandemic. Also, due to the relatively low response rate, there may have been some degree of selection bias.

\section{Conclusion}

COVID-19 has had a significant impact on the training experience of residents and fellows, and this impact will be long-lasting. As a result of the pandemic, programs at our institution converted education online, held online social events, and integrated mental health support into their programming. Programs continue to be tasked to find ways to increase educational preparedness for trainees while ensuring attention is paid to the mental well-being of the trainees. Opportunities to increase clinical learning without overwhelming trainees is a balance that all program face. Lessons from this pandemic should be used to glean ways to improve training and education for residents in the event of another pandemic or restriction of in-person learning.

\section{Abbreviations \\ CMMS: Centers for Medicare and Medicaid Services; OR: Operating Room; ACGME: Accreditation Council for Graduate Medical Education; IUH: Indiana University Health; IUSOM: Indiana University School of Medicine; GME: Gradu- ate Medical Education; ITE: In- training exam; IRB: Institutional Review Board; PPE: Personal Protective Equipment; ABS: American Board of Surgeons.}

\section{Acknowledgements}

The Indiana University GME for providing information and data and distributing the survey.

\section{Statistical methods}

All methods were performed in accordance with relevant guidelines and regulations. Spss 27 was used for all statistical methods.

\section{Authors' contributions}

S.E conceptualized the study, helped create the survey, and wrote and edited the manuscript. K.M. analyzed the data, wrote and edited the manuscript. K. S compile the data, wrote and edited the manuscript. T. I analyzed the data, created the tables, wrote and edited the manuscript. CR submitted initial study to the IRB, wrote and edited the manuscript. The author(s) read and approved the final manuscript.

\section{Funding}

No funding source was provided for this study.

\section{Availability of data and materials}

The datasets used and/or analyzed during the current study are available from the corresponding author upon request.

\section{Declarations}

\section{Ethics approval and consent to participate}

The Indiana University Institutional Review Board approved this study as an exempt status.

All guidelines and regulations regarding human participants were followed. Consent was not required per the IRB exemption. No identifiable information was obtained. Informed consent was provided prior to starting survey and clicking on the survey was regarded as consent by all survey participants.

\section{Consent for publication}

N/A

\section{Competing interests}

The authors declare they have no competing interest. 


\section{Author details}

${ }^{1}$ Division of Adolescent Medicine, Department of Pediatrics, Indiana University School of Medicine, 410 West 10th Street. Suite 1001, Indianapolis, IN 46202, USA. ${ }^{2}$ Department of Pediatrics, Indiana University School of Medicine, Indianapolis, IN, USA. ${ }^{3}$ Department of Psychiatry, Indiana University School of Medicine, Indianapolis, IN, USA. ${ }^{4}$ Indiana University School of Social Work, Indianapolis, IN, USA

Received: 10 July 2021 Accepted: 22 October 2021

Published online: 16 November 2021

\section{References}

1. Dedeilia A, Sotiropoulos MG, Hanrahan JG, Janga D, Dedeilias P, Sideris M. Medical and surgical education challenges and innovations in the COVID-19 era: a systematic review. In Vivo. 2020;34(3 suppl):1603-11.

2. O'Brien M, McNicholas F. The use of telepsychiatry during COVID-19 and beyond. Irish J Psychol Med. 2020;37(4):250-5.

3. Kannampallil TG, Goss CW, Evanoff BA, Strickland JR, McAlister RP, Duncan J. Exposure to COVID-19 patients increases physician trainee stress and burnout. PLoS One. 2020;15(8):e0237301.

4. Shrestha B, Alhafidh O, Mukhtar O, et al. Impact of COVID19 on resident physicians of a community hospital in New York city. J Community Hospital Internal Med Perspectives. 2021;11(1):4-8.

5. Civantos AM, Byrnes Y, Chang C, et al. Mental health among otolaryngology resident and attending physicians during the COVID-19 pandemic: National study. Head Neck. 2020;42(7):1597-609.

6. Indiana History. Indiana Web site. https://www.history.com/topics/usstates/indiana. Published 2018. Accessed 15 Mar 2021.

7. Indiana COVID-19 Data Report. Indiana COVID-19 Dashboard and Map.. https://www.coronavirus.in.gov/2393.htm. Published 2021. Accessed 15 Mar 2021.

8. Bean M. https://www.beckershospitalreview.com/public-health/us-coron avirus-deaths-by-state-july-1.html. Published COVID-19 Death Rate by State. Becker's Hospital Review Accessed 15 Mar 2021.

9. Health IU. Welcome to IU Health https://iuhealth.org/about-our-system. Published 2021. Accessed 15 Mar 2021.
10. Medicine IUSo. Graduate Medical Education. https://medicine.iu.edu/ gme. Published 2021. Accessed 15 Mar 2021.

11. Raj KS. Well-being in residency: a systematic review. J Grad Med Educ. 2016;8(5):674.

12. Brar B, Bayoumy M, Salama A, Henry A, Chigurupati R. A survey assessing the early effects of COVID-19 pandemic on oral and maxillofacial surgery training programs. Oral Surg Oral Med Oral Pathol Oral Radiol. 2021;131(1):27-42.

13. Rana T, Hackett C, Quezada T, et al. Medicine and surgery residents' perspectives on the impact of COVID-19 on graduate medical education. Med Educ Online. 2020;25(1):1818439.

14. Lou SS, Goss CW, Evanoff BA, Duncan JG, Kannampallil T. Risk factors associated with physician trainee concern over missed educational opportunities during the COVID-19 pandemic. BMC Med Educ. 2021;21(1):1-7.

15. Aziz H, James T, Remulla D, et al. Effect of COVID-19 on surgical training across the United States: a national survey of general surgery residents. J Surg Educ. 2021;78(2):431-9.

16. Khusid JA, Weinstein CS, Becerra AZ, et al. Well-being and education of urology residents during the COVID-19 pandemic: results of an American national survey. Int J Clin Pract. 2020;74(9):e13559.

17. Collins C, Mahuron K, Bongiovanni T, Lancaster E, Sosa JA, Wick E. Stress and the surgical resident in the COVID-19 pandemic. J Surg Educ. 2021;78(2):422-30.

18. Viswanathan R, Myers MF, Fanous AH. Support groups and individual mental health care via video conferencing for frontline clinicians during the COVID-19 pandemic. Psychosomatics. 2020;61(5):538-43.

19. Purdy AC, de Virgilio C, Kaji AH, et al. Factors Associated With General Surgery Residents' Operative Experience During the COVID-19 Pandemic. JAMA Surg. 2021;156(8):767-74. https://doi.org/10.1001/jamasurg.2021. 1978.

20. Okland TS, Pepper J-P, Valdez TA. How do we teach surgical residents in the COVID-19 era? J Surg Educ. 2020;77(5):1005-7.

\section{Publisher's Note}

Springer Nature remains neutral with regard to jurisdictional claims in published maps and institutional affiliations.
Ready to submit your research? Choose BMC and benefit from:

- fast, convenient online submission

- thorough peer review by experienced researchers in your field

- rapid publication on acceptance

- support for research data, including large and complex data types

- gold Open Access which fosters wider collaboration and increased citations

- maximum visibility for your research: over 100M website views per year

At BMC, research is always in progress.

Learn more biomedcentral.com/submissions 